\title{
POLYNOMIALS IN A HERMITIAN ELEMENT
}

\author{
by $\mathrm{M}$. J. CRABB and C. M. McGREGOR
}

(Received 15 July, 1986)

For an element $a$ of a unital Banach algebra $A$ with dual space $A^{\prime}$, we define the numerical range $V(a)=\left\{f(a): f \in A^{\prime},\|f\|=f(1)=1\right\}$, and the numerical radius $v(a)=$ $\sup \{|z|: z \in V(a)\}$. An element $a$ is said to be Hermitian if $V(a) \subseteq \mathbb{R}$, equivalently $\|\exp (i t a)\|=1(t \in \mathbb{R})$. Under the condition $V(h) \subseteq[-1,1]$, any polynomial in $h$ attains its greatest norm in the algebra $E a[-1,1]$, generated by an element $h$ with $V(h)=[-1,1]$.

In [3] we proved that in $E a[-1,1]$ all elements $a=(i h)^{m}+\xi_{0}, \xi_{0} \in \mathbb{R}$, have $v(a)=\|a\|:$ on pages 39,44 of [3] we find $\zeta \in V\left(h^{m}\right)$ such that $\left|\zeta-\tau^{\prime}\right|=\left\|h^{m}-\tau^{\prime}\right\|$. Here we extend this to any element

$$
a=\xi_{0}+\xi_{1} i h+\ldots+\xi_{m-1}(i h)^{m-1}+(i h)^{m}
$$

where $m \geqslant 1$ and $\xi_{i} \in \mathbb{R}(i=0,1, \ldots, m-1)$. As in $[3,6]$, we represent $E a[-1,1]$ as a subalgebra of the bounded linear operators on the Banach space $X$ of entire functions $f$ such that $\|f\|=\sup \{|f(z)| / \exp (|\operatorname{Im} z|): z \in \mathbb{C}\}$ exists. If $f$ is entire with $f(z) / \exp (|z|)$ bounded, and $f(x)$ is bounded for $x \in \mathbb{R}$, then $f \in X$ and $\|f\|=\sup \{|f(x)|: x \in \mathbb{R}\}$. Then $E a[-1,1]$ is generated by $h=-i D$, where $D f(z)=f^{\prime}(z)$. We show that there is a function $e$ in $X$ corresponding to a support functional $\Phi$ of the element $a$ of (1) such that $e^{\prime 2} /\left(1-e^{2}\right)$ is rational. We have $\Phi(b)=(b e)(0)(b \in E a[-1,1])$, and $e(z)=\Phi(\exp (i z h))$, which indicates how we can identify $E a[-1,1]^{\prime}$ with $X$.

We can also consider $E a[-1,1]$ as the algebra of functions $f$ on $[-1,1]$ given by $f(t)=\sum_{k=1}^{\infty} c_{k} \exp \left(i d_{k} t\right), \quad c_{k} \in \mathbb{C}, \quad d_{k} \in \mathbb{R}, \sum\left|c_{k}\right|$ finite, with $\|f\|=\inf \sum\left|c_{k}\right|$ over such representations, i.e. a quotient of $l^{1}(\mathbb{R})([5])$. The function $h(t)=t$ corresponds to the element $h$. The element $a$ of (1) has a representation as above $\Sigma c_{k} \exp \left(i d_{k} h\right)$ with $\Sigma\left|c_{k}\right|=\|a\|$, where $e\left(d_{k}\right)= \pm 1$ for all $k$. This follows from (11), and is valid for this polynomial in a Hermitian element of norm at most 1 in any Banach algebra $([1,4])$.

Note that $f \in X_{1} \Rightarrow f^{\prime} \in X_{1}$-Bernstein's inequality, or equivalently, $\|h\|=1$. Define $T \in X^{\prime}$ by $T f=(a f)(0)$, i.e.

$$
T f=\xi_{0} f(0)+\xi_{1} f^{\prime}(0)+\ldots+f^{(m)}(0) .
$$

By Lemma 4 of [3], $\|a\|=\|T\|=\sup \left\{|T f|: f \in X_{1}\right\}$, where $X_{1}=\{f \in X:\|f\| \leqslant 1\}$. This supremum is attained by an extremal function. Hence finding $\|a\|$ is equivalent to maximizing (2) over $f \in X_{1}$. R. Boas [2, Section 11.4] considers this-his method gives the extremal function when it is a translate of $\sin z$. In [3] we proved that translates of $\cos \sqrt{z^{2}+\theta^{2}}, 0 \leqslant \theta<\pi / 2$, were enough for elements $(i h)^{m}+\xi_{0}$ ([3], page 39 for $m$ even, page 41 for $m$ odd). Here we prove the following theorem.

THEOREM 1. Let $a, T$ be as in (1), (2). Then $\|a\|=\|T\|=T e$ for a certain $e \in X_{1}$

Glasgow Math. J. 30 (1988) 171-176. 
which is real on $\mathbb{R}$, and such that there exist $\mu \leqslant m-1$ and $a_{j}, b_{j} \in \mathbb{C}, a_{j} \notin \mathbb{R}, a_{j}, \bar{a}_{j} \neq b_{k}$ $(j, k=1,2, \ldots, \mu)$ such that for all $z \in \mathbb{C}$,

$$
e^{\prime 2}(z) \prod_{j=1}^{\mu}\left(z-a_{j}\right)\left(z-\bar{a}_{j}\right)=\left(1-e^{2}(z)\right) \prod_{j=1}^{\mu}\left(z-b_{j}\right)^{2} .
$$

(Allow $\mu=0$, i.e. $e^{\prime 2}=1-e^{2}$.). Further, $v(a)=\|a\|$.

We expect that the functional $T$ has an extremal function $e$ in $X_{1}$ which oscillates on $\mathbb{R}$ between \pm 1 , apart from at a finite number of turning points. $A$ theorem of Sonin-Polya [7, p. 164] pointed out by J. Duncan suggests that $e^{2}+\varphi^{-1} e^{\prime 2}=1$, where the function $\varphi$ is positive on the intervals of $\mathbb{R}$ of constant oscillation. Lemma 2 gives a sequence $p_{n}$ which converges to $e: p_{n}$ is the extremal of $T$ restricted to a class of trigonometric polynomials. Then $p_{n}^{\prime 2} /\left(1-p_{n}^{2}\right)$ is a trigonometric rational function, and a variational argument puts a bound on the number of its factors in lowest form. The Hadamard factorisations in $X$ show that the limit of this sequence, $e^{\prime 2} /\left(1-e^{2}\right)=\varphi$, is a rational function multiplied by an exponential. Using the fact that $T e$ is extremal, we prove that $\varphi$ is rational. Finally we construct $g=e+$ if $\in X_{1}$ with $f(\mathbb{R}) \subseteq \mathbb{R}$ and $|g(0)|=1$, which is enough to give $v(a)=\|a\|$.

For $n \in \mathbb{N}$, let $P_{n} \subseteq X$ be the set of functions $z \rightarrow \sum_{k=-n}^{n} \alpha_{k} \exp (i k z / n)$ where $\alpha_{k} \in \mathbb{C}$, and let $P=\bigcup_{n \in \mathbb{N}} P_{n}$.

LeMma 2. Let $f \in X_{1}$. Then there exists a sequence $\left(f_{n}\right)_{n=1}^{\infty} \subseteq P \cap X_{1}$ such that $f_{n} \rightarrow f$ as $n \rightarrow \infty$ uniformly on compact sets.

Proof. For $0<\delta<\frac{1}{2}$, define $f_{\delta}, g_{\delta} \in X$ by

$$
f_{\delta}(z)=f((1-2 \delta) z)(\delta z)^{-2} \sin ^{2} \delta z=g_{\delta}(z) / z^{2}
$$

Then $f_{\delta} \rightarrow f$ as $\delta \rightarrow 0$ uniformly on compact sets. Since $\left|f_{\delta}(x)\right| \leqslant 1 \quad(x \in \mathbb{R})$, we have $f_{\delta} \in X_{1}$. Thus it is enough to prove the lemma for a function $f \in X_{1}$ given by $f(z)=g(z) / z^{2}$, where $g \in X$. Given such a function, define $f_{n}(z)=\sum_{k \in \mathbb{Z}} f(z+2 k n \pi)$. $(n \in \mathbb{N})$. Since $g$ is bounded on lines $\operatorname{Im} z=$ constant, the series converges. For $|\operatorname{Re} z| \leqslant n \pi$ we have

$$
\left|f_{n}(z)-f(z)\right| \leqslant 2\|g\| \exp (|\operatorname{Im} z|) \sum_{k=1}^{\infty}(n \pi(2 k-1))^{-2}=\alpha n^{-2} \exp (|\operatorname{Im} z|),
$$

where $\alpha=\|g\| / 4$. Since $f \in X_{1}$ and $f_{n}$ has period $2 n \pi$, we deduce that

$$
\left|f_{n}(z)\right| \leqslant\left(1+\alpha n^{-2}\right) \exp (|\operatorname{Im} z|)(z \in \mathbb{C}) \text {. }
$$

Therefore $f_{n} \in X$ and $f_{n} \rightarrow f$ uniformly on compact sets as $n \rightarrow \infty$. Since $f_{n}$ has period $2 n \pi$, by [2, Theorem 6. 10.1], $f_{n} \in P_{n}$. Replacing $f_{n}$ by $\left(1+\alpha n^{-2}\right)^{-1} f_{n}$, we get the required series.

For the sequence of Lemma $2, \lim _{n \rightarrow \infty} f_{n}^{(k)}(0)=f^{(k)}(0)(k=0,1,2, \ldots)$, and so $\lim _{n \rightarrow \infty} T f_{n}=$ Tf. Hence $\|T\|=\sup \left\{|T f|: f \in P \cap \grave{X}_{1}\right\}$. Let $p_{n} \in P_{n} \cap X_{1}$ be such that

$$
T p_{n}=\sup \left\{|T f|: f \in P_{n} \cap X_{1}\right\} \text {. }
$$


Since for $p_{n}^{*}(z)=\overline{p_{n}(\bar{z})}, T p_{n}^{*}=\overline{T p_{n}}=T p_{n}$, by replacing $p_{n}$ by $\frac{1}{2}\left(p_{n}+p_{n}^{*}\right)$ we can assume that $p_{n}$ is real-valued on $\mathbb{R}$. Since $X_{1}$ is a normal family, there is a subsequence $\left(p_{n_{j}}\right)$ such that $\lim _{j \rightarrow \infty} T p_{n_{j}}=\|T\|$ and $\lim _{j \rightarrow \infty} p_{n_{j}}=e \in X_{1}$, with uniform convergence on compact sets.

Therefore $\lim _{j \rightarrow \infty} T p_{n_{j}}=T e=\|T\|$, and $e$ is an extremal function for $T$.

If $e^{\prime}(0)=e^{\prime \prime}(0)=\ldots=e^{(m)}(0)=0$, then $T e=T e_{1}$ where $e_{1}$ is a constant function, which satisfies (3) for $\mu=0$. Henceforth we assume that one of $e^{\prime}(0), \ldots, e^{(m)}(0)$ is non-zero. By taking a further subsequence, we can assume that one of $p_{n_{j}}^{\prime}(0), \ldots, p_{n_{j}}^{(m)}(0)$ is non-zero for each $j$.

LeMMA 3. If $p_{n}+i q_{n}$ (resp. $\left.e+i f\right) \in X_{1}$ for some $q_{n} \in P_{n}$ (resp. $\left.f \in X\right)$ with $q_{n}(\mathbb{R}) \subset \mathbb{R}$ $($ resp. $f(\mathbb{R}) \subset \mathbb{R})$, then $T q_{n}=0($ resp. $T f=0)$.

Proof. By (4), $\left|T p_{n}+i T q_{n}\right|=\left|T\left(p_{n}+i q_{n}\right)\right| \leqslant T p_{n}$. Since $T p_{n}, T q_{n} \in \mathbb{R}$, we get $T q_{n}=0$. The second part is similar.

Lemma 4. Let a, e be as in Theorem 1. Suppose that there exists $f \in X$, real on $\mathbb{R}$, such that $g=e+i f \in X_{1}$ and $|g(0)|=1$. Then $v(a)=\|a\|$.

Proof. By Lemma 3, Tf $=0$. Put $g_{1}=\overline{g(0)} g$, so that $g_{1} \in X_{1}$ and $g_{1}(0)=1$. By Lemma 1 of [3], $T g_{1}=\left(a g_{1}\right)(0) \in V(a)$. Hence $v(a) \leqslant\|a\|=T e=T g=\left|T g_{1}\right| \leqslant v(a)$, where the inequalities follow from the definition of $v(a)$.

Proof of Theorem 1. If $f \in P_{n}$ is real on $\mathbb{R}$, so that for some $v, f(z)=$ $\sum_{k=-v}^{v} a_{k} \exp (i k z / n)$ with $a_{v} \neq 0$, we prove later that we may write

$$
f(z)=\lambda \prod_{k=1}^{2 v} \sin \left(\left(z-z_{k}\right) / 2 n\right)(z \in \mathbb{C}),
$$

where $\lambda \in \mathbb{R}$ and we may assume that $-n \pi<\operatorname{Re} z_{k} \leqslant n \pi$. For $p=p_{n_{j}}$ as in (4), we factorize $p^{\prime}$ and $1 \pm p$ : the same ' $v$ ' appears for each function. Since $p^{\prime 2}$ and $1-p^{2}$ are non-negative on $\mathbb{R}$ we get for some $\lambda>0,-n \pi<\operatorname{Re} \alpha_{k}, \operatorname{Re} \beta_{k} \leqslant n \pi$, where we write $n$ for $n_{j}$,

$$
p^{\prime 2}(z)\left(1-p^{2}(z)\right)^{-1}=\lambda^{2} \prod_{k=1}^{2 v} \sin ^{2}\left(\left(z-\beta_{k}\right) / 2 n\right) / \prod_{k=1}^{4 v} \sin \left(\left(z-\alpha_{k}\right) / 2 n\right) .
$$

If $\alpha_{k} \in \mathbb{R}$, then $p\left(\alpha_{k}\right)= \pm 1$, which gives $p^{\prime}\left(\alpha_{k}\right)=0$, and we find that $p^{\prime 2}$ has a zero of order at least that of $\left(1-p^{2}\right)$ at $\alpha_{k}$. Hence by cancellation we can assume in (5) that $\alpha_{k} \notin \mathbb{R}$, and by similar reasoning that $\alpha_{k} \neq \beta_{j}$, for all $j, k$. The $\alpha_{k}$ are in complex conjugate pairs. Hence for some $\mu$, for all $z \in \mathbb{C}$,

$$
p^{\prime 2}(z)\left(1-p^{2}(z)\right)^{-1}=\lambda^{2} \prod_{k=1}^{\mu} \sin ^{2}\left(\left(z-\beta_{k}\right) / 2 n\right)\left[\sin \left(\left(z-\alpha_{k}\right) / 2 n\right) \sin \left(\left(z-\bar{\alpha}_{k}\right) / 2 n\right)\right]^{-1} .
$$

Write $q(z)=\lambda^{-1} p^{\prime}(z) / \prod_{k=1}^{\mu} \sin \left(\left(z-\beta_{k}\right) / 2 n\right)$. Thus

$$
q(z)=(\text { constant }) \prod_{k=\mu+1}^{2 v} \sin \left(\left(z-\beta_{k}\right) / 2 n\right),
$$


from (5). By (6),

$$
p^{2}(z)+q^{2}(z) \prod_{k=1}^{\mu} \sin \left(\left(z-\alpha_{k}\right) / 2 n\right) \sin \left(\left(z-\bar{\alpha}_{k}\right) / 2 n\right)=1 \quad(z \in \mathbb{C}) .
$$

The function $x \rightarrow \prod_{k=1}^{\mu}\left|\sin \left(\left(x-\alpha_{k}\right) / 2 n\right)\right|^{2}$ is continuous, periodic and non-zero on $\mathbb{R}$, and hence is bounded below by some $\delta^{2}>0$. Hence by (7), $p^{2}(x)+\delta^{2} q^{2}(x) \leqslant 1(x \in \mathbb{R})$, and so $P_{n} \cap X_{1}$ contains the function

$$
x \rightarrow p(x)+i \delta q(x) \sin ^{j}(x / 2 n) \cos ^{\mu-j}(x / 2 n), \quad(j=0,1,2, \ldots, \mu) .
$$

Since one of $p^{\prime}(0), \ldots, p^{(m)}(0)$ is non-zero, from the definition of $q$, (at least) one of $q(0), q^{\prime}(0), \ldots, q^{(m-1)}(0)$ is non-zero.

Suppose if possible that $\mu \geqslant m$. Choose $j \leqslant m$ such that $q^{(m-j)}(0) \neq 0$ and $q^{(k)}(0)=0$ $(k<m-j)$. By Lemma 3 and (2) we have

$$
0=T\left(q(z) \sin ^{j}(z / 2 n) \cos ^{\mu-j}(z / 2 n)\right)=m ! q^{(m-j)}(0) /\left((m-j) !(2 n)^{j}\right):
$$

the function to which $T$ is applied has a leading term in $z^{m}$. Hence $\mu \leqslant m-1$.

By Hadamard's factorization theorem, any $f \in X$ can be written

$$
f(z)=\beta \exp (\alpha z) z^{\lambda} \prod_{k=1}^{\infty}\left(1-a_{k}^{-1} z\right) \exp \left(z / a_{k}\right),
$$

where $a_{k}$ are the zeros. By (6) each function $p_{n_{j}}^{\prime 2}, 1-p_{n_{j}}^{2}$ has, counting multiplicity, at most $2(m-1)$ zeros in $-n_{j} \pi<\operatorname{Re} z \leqslant n_{j} \pi$ which the other does not have. Each zero of $e^{\prime 2},\left(1-e^{2}\right)$ is a limit of zeros of $p_{n_{j}}^{\prime 2},\left(1-p_{n_{j}}^{2}\right)$. Therefore the factorizations of $e^{\prime 2}$ and $1+e, 1-e$ give, where $\alpha, \beta \in \mathbb{R}, \beta>0$ (since $e^{\prime 2}$ and $1-e^{2}$ are non-negative on $\mathbb{R}$ ) and $\mu_{1}, \mu_{2} \leqslant m-1, a_{k}, b_{k} \in \mathbb{C}$,

$$
\varphi(z)=e^{\prime 2}(z)\left(1-e^{2}(z)\right)^{-1}=\beta \exp (\alpha z) \prod_{k=1}^{\mu_{1}}\left(z-b_{k}\right)^{2} \prod_{k=1}^{\mu_{2}}\left(z-a_{k}\right)^{-1}\left(z-\bar{a}_{k}\right)^{-1},
$$

where we are defining $\varphi$. By the same argument as for $p$, we can assume that $a_{k} \notin \mathbb{R}$ and $a_{k}, \bar{a}_{k} \neq b_{j}$ for all $j, k$.

Since $e$ is not constant, there is a disc $\Delta=\{|z-\xi|<\eta\}, \xi \in \mathbb{R}$, on which $|e|<1$. On $\Delta, \sigma(z)=\sin ^{-1}(e(z))$ is analytic. For $z \in \Delta \cap \mathbb{B}, \sigma^{\prime}(z)=e^{\prime}(z)\left(1-e^{2}(z)\right)^{-1 / 2}=\psi(z)$, where by $(8)$,

$$
\psi(z)= \pm \beta^{1 / 2} \exp \left(\frac{1}{2} \alpha z\right) \prod_{k=1}^{\mu_{1}}\left(z-b_{k}\right) / \prod_{k=1}^{\mu_{2}}\left[\left(z-a_{k}\right)\left(z-\bar{a}_{k}\right)\right]^{1 / 2} .
$$

Since $\left(z-a_{k}\right)\left(z-\bar{a}_{k}\right)>0$, we have the usual square root here. Hence on $\Delta \cap \mathbb{R}$, for some $\theta \in \mathbb{R}$ we have

$$
e(z)=\sin \left(\theta+\int_{\xi}^{z} \psi(t) d t\right)
$$


The right-hand side of (9) defines a function analytic in a neighbourhood of $\mathbb{R}$, which must therefore equal $e$.

Suppose if possible that $|\psi|>1$ for all $t>$ (or $<$ ) some $t_{0} \in \mathbb{R}$. Then by (9) for certain $x_{n} \in \mathbb{R}$ with $\left|x_{n}\right| \rightarrow \infty$ as $n \rightarrow \infty$, we have $e\left(x_{n}\right)=0$ and, by $(8), e^{\prime}\left(x_{n}\right)^{2}=\varphi\left(x_{n}\right)=\psi^{2}\left(x_{n}\right)>$ 1. This contradicts Bernstein's inequality $\left|e^{\prime}(x)\right| \leqslant 1(x \in \mathbb{R})$. Therefore in (8), $\alpha=0$ and $\mu_{2} \geqslant \mu_{1}$. Hence by $(8), \varphi(x) \leqslant K(x \in \mathbb{R})$ for some $K>0$, and $e^{2}(x)+K^{-1} e^{\prime 2}(x) \leqslant 1$ $(x \in \mathbb{R})$.

Equation (9) holds for any $z$ if we integrate along a curve not through any $a_{k}$ or $\bar{a}_{k}$, and take the square root continuously in $\psi$. If $|\psi| \leqslant \delta<1$ for all large $|z|$, then (9) shows that $e^{\prime}(z) / \exp (\delta|z|)$ is bounded on $\mathbb{C}$. Hence if $\gamma>0, j \in \mathbb{N}$ and $\delta+j \gamma \leqslant 1$, then $e^{\prime}(z) \sin ^{j} \gamma z \in X$, and $e(z)+i K^{-1 / 2} e^{\prime}(z) \sin ^{j} \gamma z \in X_{1}$. Choose $\gamma, j$ such that $e^{(m-j+1)}(0) \neq 0$ and $e^{(k)}(0)=0(1 \leqslant k \leqslant m-j)$. Then by Lemma 3 and $(2), 0=T\left(e^{\prime}(z) \sin ^{j} \gamma z\right) \neq 0$. Thus $|\psi| \leqslant \delta<1$ for all large $|z|$ is not possible, which gives $\mu_{2}=\mu_{1}$ and $\beta \geqslant 1$. Since $\beta>1$ would give $|\psi|>1$ for all large real $x$, we have $\beta=1$. Equation (8) now gives us (3).

Define $\sigma_{k}(z)=\left|a_{k}\right|-\left|a_{k}\right|^{-1}\left(\operatorname{Re} a_{k}\right) z \quad(z \in \mathbb{C}, k=1,2, \ldots, \mu)$. For $t \in \mathbb{R}$, we have $\sigma_{k}^{2}(t) \leqslant\left(t-a_{k}\right)\left(t-\bar{a}_{k}\right)$, with equality when $t=0$. Then (3) gives

with equality when $t=0$.

$$
e^{2}(t)+e^{\prime 2}(t) \prod_{k=1}^{\mu} \frac{\sigma_{k}^{2}(t)}{\left(t-b_{k}\right)^{2}} \leqslant 1 \quad(t \in \mathbb{R})
$$

Since in (3) $a_{j}, \bar{a}_{j} \neq b_{k}$, it follows that $e^{\prime}(z) / \prod_{j=1}^{\mu}\left(z-b_{j}\right)$ is entire. Hence $f(z)=$ $e^{\prime}(z) \prod_{j=1}^{\mu} \sigma_{j}(z)\left(z-b_{j}\right)^{-1} \in X$, since $e^{\prime} \in X$ and $\prod_{j=1}^{\mu} \sigma_{j}(z)\left(z-b_{j}\right)^{-1}$ tends to a limit as $|z| \rightarrow \infty$. By $(10)$ and since $f(\mathbb{R}) \subseteq \mathbb{R},|(e+i f)(x)| \leqslant 1(x \in \mathbb{R})$, and so $e+i f \in X_{1}$. The case $t=0$ in (10) gives $|(e+i f)(0)|=1$. By Lemma $4, v(a)=\|a\|$.

To derive the factorization of a function in $P_{n}$ given, use the Hadamard factorization, grouping the zeros in subsets of period $2 n \pi$. If the function is real on $\mathbb{R}$, there is no factor $\exp (\beta z)$ "left over". This completes the proof of Theorem 1.

Remarks. For each $m$ there exist elements $a$ such that in (3) we have $\mu=m-1$. For example, let $\xi_{i} \in \mathbb{C}$ be such that the element $a$ of (1) has minimum norm. We can show that in fact $\xi_{i} \in \mathbb{R}$. There exists $\Phi \in A^{\prime}$ such that $\|\Phi\|=1, \Phi(a)=\|a\|$, and $\Phi\left(h^{j}\right)=0$ $(0 \leqslant j<m)$. The function $e(z)=\Phi(\exp (i z h))$ is an extremal for $a$, with $e^{(j)}(0)=0$ $(0 \leqslant j<m)$. If we replace $e$ by $\frac{1}{2}\left(e+e^{*}\right)$, (3) becomes

$$
e^{\prime 2}(z) \prod_{k=1}^{m-1}\left(z-a_{k}\right)\left(z-\bar{a}_{k}\right)=z^{2 m-2}\left(1-e^{2}(z)\right) .
$$

In the case $m=3$, this $a$ is $-i\left(h^{3}-\xi_{1} h\right)$, and (9) becomes, for a certain $\alpha$,

$$
e(z)=\sin \int_{0}^{z} \psi(t) d t, \quad \text { where } \quad \psi(t)=t^{2} /[(t-\alpha)(t+\alpha)(t-\bar{\alpha})(t+\bar{\alpha})]^{1 / 2} .
$$

We require $\int_{0}^{\alpha} \psi= \pm \pi / 2$, and calculation with elliptic functions gives $\xi_{1} \bumpeq 0.73, \alpha \bumpeq$ $0.97+i 2.10,\left\|h^{3}-\xi_{1} h\right\| \bumpeq 0.37$.

We can prove (omitted) the following necessary and sufficient condition that a given 
function $e \in X_{1}$, real on $\mathbb{R}$, is the extremal for $T$ as in (2): for $a, T$ given, $e$ is in fact unique, and not constant.

(i) $e$ satisfies an equation of the form of (3), and

(ii) there exist sequences $c_{k}, d_{k} \in \mathbb{R}$ such that for any $f \in X$, the contour integral

$$
\int_{\Gamma} T_{w}\left(\frac{e^{\prime}(w)}{w-z}\right) \frac{f(z)}{e^{\prime}(z)} d z
$$

where $T_{w}$ denotes $T$ with the differentiations carried out with respect to $w$, and contours $\Gamma \rightarrow \infty$, gives as the sum of the residues of the integrand (which equals 0 ) a fixed multiple of $T f-\sum_{k} c_{k} f\left(d_{k}\right)$. Further, $c_{k} e\left(d_{k}\right) \geqslant 0$ for all $k$.

For $\mu=1$ in (3), $e$ is a translate of $\cos \sqrt{z^{2}+\theta^{2}}, 0 \leqslant \theta$.

The functions found here are also the extremals for operators of the form $T f=\sum_{j=1}^{n} \beta_{j} f\left(\alpha_{j}\right)$, where $\alpha_{j}, \beta_{j} \in \mathbb{R}$, and we require the maximum over $f \in X_{1}:$ this $T$ has an extremal $e$ with $\mu \leqslant m-2$ in (3). Boas [2, Theorem 11.4.1] gives this result for $T f=f(\delta)-f(-\delta)$.

From (3), if $e^{\prime}(z)=0$ then, with at most $(m-1)$ exceptions, $e(z)= \pm 1$. Thus on $\mathbb{R}$, outside the interval spanned by the $b_{j}$ 's which happen to be real, $e$ oscillates between \pm 1 .

\section{REFERENCES}

1. M. Baillet, Un calcul fonctionnel de class $C^{1}$ pour les operateurs pre-hermitiens d'un espace de Banach, C.R. Acad. Sci. Paris, Ser A, 283 (1976), 891-894.

2. R. P. Boas, Entire functions (Academic Press, 1954).

3. M. J. Crabb and C. M. McGregor, Numerical ranges of powers of Hermitian elements, Glasgow Math. J. 28 (1986), 37-45.

4. H. Konig, A functional calculus for Hermitian elements of complex Banach algebras, Archiv der Math. 28 (1977), 422-430.

5. A. M. Sinclair, The Banach algebra generated by a Hermitian operator, Proc. London Math: Soc. (3) 24 (1972), 681-691.

6. A. M. Sinclair, The Banach algebra generated by a derivation, Operator Theory: Adv. Appl. 14 (1984), 241-250.

7. G. Szego, Orthogonal polynomials, American Math. Soc. Coll. Publ. XXIII (1959).

DepartMENT OF MATHEMATICS

UNIVERSITY OF GLASGOW 